\title{
Cardiovascular and neuropsychiatric risks of varenicline: too good to be true? Reply
}

Citation for published version (APA):

Kotz, D., Viechtbauer, W., Simpson, C., van Schayck, O. C. P., West, R., \& Sheikh, A. (2015).

Cardiovascular and neuropsychiatric risks of varenicline: too good to be true? Reply. The Lancet Respiratory medicine, 3(12), E40-E42. https://doi.org/10.1016/S2213-2600(15)00470-1

Document status and date:

Published: 01/12/2015

DOI:

10.1016/S2213-2600(15)00470-1

Document Version:

Publisher's PDF, also known as Version of record

Document license:

Taverne

Please check the document version of this publication:

- A submitted manuscript is the version of the article upon submission and before peer-review. There can be important differences between the submitted version and the official published version of record.

People interested in the research are advised to contact the author for the final version of the publication, or visit the DOI to the publisher's website.

- The final author version and the galley proof are versions of the publication after peer review.

- The final published version features the final layout of the paper including the volume, issue and page numbers.

Link to publication

\footnotetext{
General rights rights.

- You may freely distribute the URL identifying the publication in the public portal. please follow below link for the End User Agreement:

www.umlib.nl/taverne-license

Take down policy

If you believe that this document breaches copyright please contact us at:

repository@maastrichtuniversity.nl

providing details and we will investigate your claim.
}

Copyright and moral rights for the publications made accessible in the public portal are retained by the authors and/or other copyright owners and it is a condition of accessing publications that users recognise and abide by the legal requirements associated with these

- Users may download and print one copy of any publication from the public portal for the purpose of private study or research.

- You may not further distribute the material or use it for any profit-making activity or commercial gain

If the publication is distributed under the terms of Article $25 \mathrm{fa}$ of the Dutch Copyright Act, indicated by the "Taverne" license above, 
patients prescribed varenicline were at lower risk of depression and selfharm. Additionally, our observational study ${ }^{5}$ found that patients prescribed varenicline had substantially lower mortality compared with patients prescribed nicotine replacement therapy. By contrast, the meta-analysis of randomised controlled trials ${ }^{4}$ identified little evidence that patients prescribed varenicline were less likely to be depressed, self-harm, or to die. If the adjusted observational results are biased for depression and self-harm, it is probable that they are biased for the other outcomes as well.

We can only interpret Kotz and colleagues' results as unbiased estimates of the causal effects of varenicline if we assume that there are no unmeasured confounders. This assumption is unlikely to hold. While Kotz and colleagues present the results of a simulation of the effects of a single unmeasured binary confounder, the true confounding structure is likely to be much more complex, involving multiple clustered confounders, which could produce the observed results with far smaller effects than assumed in the simulation.

Observational studies can only produce credible evidence of the causal effects of an exposure if they propose a robust method of overcoming residual or unmeasured confounding. One such approach is instrumental variable analysis, which attempts to mimic randomisation and allows equal distribution of measured and unmeasured confounders. ${ }^{6}$ It relies on the availability of an instrument that is associated with the exposure, is not associated with the confounding factors, and has no direct effect on the outcome of interest. In their published protocol ${ }^{7}$ Kotz and colleagues proposed the use of an instrumental variable analysis, but did not include this in their final report because of the difficulty in identifying valid instruments. Physicians' prescribing preferences, identified on the basis of physicians' previous prescriptions, have been shown to be potentially valid instruments for a number of medications including smoking cessation therapies. ${ }^{5,8,9}$ With use of instrumental variable methods we have identified no evidence that patients prescribed varenicline had a decreased risk of depression, self-harm, or death compared with those prescribed nicotine replacement therapy. ${ }^{4}$ This suggests that the reductions in the risk of outcomes suggested by conventional analyses in the observational studies, 2,5 including the large protective effect on all-cause mortality, are likely to be due to residual confounding.

We broadly agree with Kotz and colleagues' conclusions regarding the safety of varenicline. The results from the Food and Drug Administration's adverse Event Reporting System database, which suggested patients prescribed varenicline have an increased risk of depression and suicidal behaviour, are likely to be biased by residual confounding. ${ }^{10}$ Regulators such as the Food and Drug Administration have used this evidence to justify imposing safety warnings on varenicline, but these safety warnings are increasingly inconsistent with evidence from several sources. Research into the safety of varenicline should use robust methods to try to overcome problems of bias. Epidemiologists are acutely aware that large observational studies can produce very precise, but very biased evidence, ${ }^{1}$ but the public and policy makers might not be.

AET has received a grant from the Global Research Awards for Nicotine Dependence (GRAND) which is supported by Pfizer. All other authors declare no competing interests.

*Neil M Davies, Gemma Taylor, Amy E Taylor, Richard M Martin, Marcus R Munafò, Kyla HThomas neil.davies@bristol.ac.uk

Medical Research Council Integrative Epidemiology Unit, University of Bristol, BS8 2BN, UK (NMD, GT, AET, RMM, MRM); School of Social and Community Medicine, University of Bristol, Barley House, Oakfield Grove, Bristol, UK (NMD, GT, RMM, KHT); School of Experimental Psychology, University of Bristol, Bristol, UK (AET, MRM)
1 Davey-Smith G, Ebrahim S. Epidemiology-is it time to call it a day? Int J Epidemiol 2001; 30: 1.

2 Kotz D, Viechtbauer W, Simpson C, van Schayck OCP, West R, Sheikh A Cardiovascular and neuropsychiatric risks of varenicline: a retrospective cohort study. Lancet Respir Med 2015; 3: 761-68.

3 Bulik-Sullivan BK, Loh P-R, Finucane HK, et al. LD Score regression distinguishes confounding from polygenicity in genome-wide association studies. Nat Genet 2015; 47: 291595

4 Thomas KH, Martin RM, Knipe DW, Higgins JPT, Gunnell D. Risk of neuropsychiatric adverse events associated with varenicline: systematic review and meta-analysis. BMJ 2015; 350: h1109.

5 Thomas KH, Martin RM, Davies NM, Metcalfe C, Windmeijer F, Gunnell D. Smoking cessation treatment and risk of depression, suicide, and self harm in the Clinical Practice Research Datalink: prospective cohort study. BMJ 2013; 347: f5704.

6 Hernn KH, Robins J. Instruments for causal inference: an epidemiologist's dream? Epidemiology 2006; 17: 3606.

7 Kotz D, Simpson C, Viechtbauer W, van Schayck OCP, West R, Sheikh A. Cardiovascular and neuropsychiatric safety of varenicline and bupropion compared with nicotine replacement therapy for smoking cessation: study protocol of a retrospective cohort study using the QResearch general practice database. BMJ Open 2014; 4: e005281.

8 Davies NM, Gunnell D, Thomas KH, Metcalfe C, Windmeijer F, Martin RM. Physicians' prescribing preferences were a potential instrument for patients' actual prescriptions of antidepressants. J Clin Epidemiol 2013; 66: 1386-96

9 Davies NM, Davey Smith G, Windmeijer F, Martin RM. COX-2 Selective nonsteroidal anti-inflammatory drugs and risk of gastrointestinal tract complications and myocardial infarction: an instrumental variable analysis. Epidemiology 2013; 24: 352-62.

10 Moore TJ, Furberg CD, Glenmullen J, Maltsberger JT, Singh S. Suicidal behavior and depression in smoking cessation treatments. PLoS One 2011; 6: e27016.

\section{Authors' reply}

In their letter, Foluke Odeyale and Itoro Udo make two points with respect to our investigation into the cardiovascular and neuropsychiatric risks of varenicline for smoking cessation. ${ }^{1}$ First, they mention that we did not consider neuropsychiatric events such as anxiety, agitation, psychosis, and suicidal ideation. This is because we were only able to include events that are routinely recorded by general practitioners in the UK. We addressed this limitation in the discussion section of our Article. That said, it is important to emphasise the point that we found 
no evidence to indicate an increased risk of depression and self-harm, which have been identified as among the most important serious adverse events and are included in the US Food and Drug Administration's black box warning for varenicline.

Second, Odeyale and Udo suggest that we should have included "previous secondary mental health care and admission" as a potential confounder in our analyses. We would like to make two points in response. What they speculate about is not an example of confounding, but a potential interaction between the use of varenicline and previous secondary mental health-care treatment. The purpose of our study, however, was to investigate the risks of varenicline in the general population of smokers rather than in a specific subgroup. Therefore, our large, non-restrictive sample included smokers with previous mental illness. Also, we did include key markers of mental illness as confounding variables and, uniquely, modelled what would need to be the distribution and effect of any unmeasured confounders to invalidate our key conclusions. We showed that such confounders would only lead to an increased risk associated with varenicline use under very unlikely assumptions.

In another correspondence, Johannes Nielen and Andrea Burden comment on our finding that patients with a prescription of nicotine replacement therapy showed a higher prevalence of previous cardiovascular and neuropsychiatric events than patients with a prescription of varenicline or bupropion. They offer the same explanation as we did in the discussion section of our Article ${ }^{1}$-ie, that health-care professionals seem less likely to prescribe varenicline and bupropion to patients with smokingrelated illnesses.

Our interesting and important finding was only made possible by using "real-world" observational data from one of the largest general practice databases in the world. An advantage of such data is the high generalisability of findings because-contrary to randomised controlled trials-all patients, including those with pre-existing cardiovascular and neuropsychiatric disease and co-morbidities, are included. Such patients, however, seem to be more likely to receive a prescription of nicotine replacement therapy. In our statistical analyses, we therefore used adjusted multivariable regression modelling and propensity score analysis to account for confounding due to these differences. Details about propensity score analysis are provided in the Article's appendix and our published protocol. ${ }^{1,2}$ We agree with Nielen and Burden that these two methods cannot fully account for residual confounding; however, the authors seem to have missed the fact that we additionally modelled potential unmeasured confounders and were able to show that an increased risk of any of the neuropsychiatric and cardiovascular events assessed in varenicline users was very unlikely. On the basis of our extensive analyses and the evidence from several other studies, ${ }^{3-7}$ we are assured that our key conclusions are correct.

Furthermore, we agree with the general point made by Davies and colleagues that research on the safety of varenicline that uses observational data should use robust methods to account for confounding. In our retrospective cohort study, ${ }^{1}$ we therefore used multivariable regression modelling with adjustment for an extensive list of potential confounders and propensity score analysis.

Instrumental variable analysis was a third analytical approach we had planned, as stated in our published protocol. ${ }^{2}$ In theory, an instrumental variable resembles the tossing of a coin to assign patients to a treatment in randomised controlled trials and so becomes a powerful method to overcome unmeasured confounding. In practice, however, it is generally very difficult to identify a valid and strong instrumental variable. Providers of health-care databases that are used for research should therefore consider preparing their data in such a way that they fit the purpose of an instrumental variable analysis and ideally help with identifying instrumental variables for a particular study.

We were not able to identify an instrumental variable for our study so, instead, we modelled-for the first time in this context-what the effect and distribution of any potential unmeasured confounders needed to be to reverse our findings and to show an increased risk associated with varenicline use. With this modelling, we were able to show that an increased risk of any of the neuropsychiatric and cardiovascular events assessed in varenicline users was very unlikely.

Reassuringly, in their own observational study into the risk of depression, suicide, and self-harm in varenicline users, Davies and colleagues identified no difference between the conventional regression modelling and the instrumental variable analysis, and came to the same conclusion as we did. ${ }^{8}$ Taken together, we hope that these carefully undertaken, large-scale observational studies ${ }^{1,8}$ will lead decision makers-such as the US Food and Drug Administration-to review their safety warnings for varenicline.

The authors' competing interests remain the same as those declared in the original article.

*Daniel Kotz, Wolfgang Viechtbauer, Colin Simpson, Onno C P van Schayck, Robert West, Aziz Sheikh

\section{daniel.kotz@med.uni-duesseldorf.de}

Institute of General Practice, Medical Faculty of the Heinrich-Heine-University Düsseldorf, 40225 Düsseldorf, Germany (DK); Department of Family Medicine, CAPHRI School for Public Health and Primary Care, Maastricht University Medical Centre,

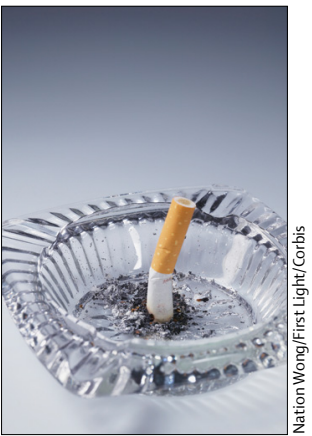


Maastricht, the Netherlands (DK, OCPvS, AS); Allergy \& Respiratory Research Group, Centre for Medical Informatics, Usher Institute of Population Health Sciences and Informatics, The University of Edinburgh, Edinburgh, UK (DK, OCPvS, AS, CS); Cancer Research UK Health Behaviour Research Centre, University College London, London, UK (DK, RW); MHeNS School for Mental Health and Neuroscience, Maastricht University, Maastricht Netherlands (WV); and Division of General Internal Medicine and Primary Care, Brigham and Women's Hospital, Harvard Medical School, Boston, MA, USA (AS)

1 Kotz D, Viechtbauer W, Simpson C van Schayck OC, West R, Sheikh A. Cardiovascular and neuropsychiatric risks of varenicline: a retrospective cohort study. Lancet Respir Med 2015; 3: 761-68.

2 Kotz D, Simpson C, Viechtbauer W, van Schayck OCP, West R, Sheikh A Cardiovascular and neuropsychiatric safety of varenicline and bupropion compared with nicotine replacement therapy for smoking cessation: study protocol of a retrospective cohort study using the QResearch general practice database. BMJ Open 2014; 4: e005281.

3 Prochaska JJ, Hilton JF. Risk of cardiovascular serious adverse events associated with varenicline use for tobacco cessation: systematic review and meta-analysis. BM 2012; 344: e2856

4 Cahill K, Stevens S, Lancaster T. Pharmacological treatments for smoking cessation. JAMA 2014; 311: 193-94.

5 Mills El, Thorlund K, Eapen S, Wu P, Prochaska JJ. Cardiovascular events associated with smoking cessation pharmacotherapies: a network meta-analysis. Circulation 2014; 129: $28-41$

6 Hughes JR. Varenicline as a cause of suicidal outcomes. Nicotine Tob Res 2015; published online Jan 8. DOI: 10.1093/ntr/ntu275.

7 Pfizer. Briefing document. Joint meeting of the psychopharmacologic drugs advisory committee and drug safety and risk management advisory committee. Risk of serious neuropsychiatric adverse events with chantix (varenicline tartrate). http://www.fda gov/downloads/AdvisoryCommittees/ CommitteesMeetingMaterials/Drugs/ PsychopharmacologicDrugsAdvisory Committee/UCM418706.pdf (accessed Nov 6, 2015)

8 Thomas KH, Martin RM, Davies NM, Metcalfe C, Windmeijer F, Gunnell D. Smoking cessation treatment and risk of depression, suicide, and self harm in the Clinical Practice Research Datalink: prospective cohort study. BMJ 2013; 347: 1-9.

\section{Use of $\mathrm{FEV}_{1}$ as a measure of lung health in the UK BiLEVE study}

We read with interest the Article by Louise Wain and colleagues, ${ }^{1}$ but we were puzzled by the choice of $\mathrm{FEV}_{1}$ as the respiratory outcome to represent lung health. $\mathrm{FEV}_{1}$ in isolation is rarely used as a clinical measurement because it is affected by many factors unrelated to pulmonary function, such as effort and total lung volume. Although the authors attempted to account for some of these factors by calculating a "predicted $\mathrm{FEV}_{1}$ " for bands of age and sex, regressing $\mathrm{FEV}_{1}$ by height, and defining the reference group as healthy never-smokers, the usual practice, in interpretation of pulmonary function, is to examine the ratio of FEV ${ }_{1}$ to FVC. Without FVC data, it is difficult to know whether the "high $\mathrm{FEV}_{1}$ " group really does have normal lung function.

The use of a non-standard measurement of pulmonary function when standard measures are readily available is especially puzzling because the authors apparently have access to FVC measurements in their participants. These issues are further amplified by the absence of information about how the lung volumes were measured, how the equipment was standardised and calibrated, and by the absence of absolute values.

The very small amount of variance (4\%) accounted for either by the previously identified (2\%) or by the newly identified (1\%) genetic variants seems to be within the ranges of test-retest values and calibration measurements of even the best pulmonary function laboratories. The small fraction of the variance explained by the new variants, in concert with the method considerations mentioned, only serve to fuel skepticism about the often promised ultimate clinical usefulness of large scale sequencing efforts for common diseases.

\section{We declare no competing interests. \\ *David O Warner, Michael J Joyner, Nigel Paneth \\ warner.david@mayo.edu}

Department of Anesthesiology, Mayo Clinic Rochester, MN 55905, USA (DOW, MJJ); and Departments of Epidemiology and Biostatistics and Pediatrics and Human Development, Michigan State University, MI, USA (NP)
1 Wain LV, Shrine N, Miller S, et al. Novel insights into the genetics of smoking behaviour, lung function, and chronic obstructive pulmonary disease (UK BiLEVE): a genetic association study in UK Biobank. Lancet Respir Med 2015; 3: 769-81.

\section{Authors' reply}

We thank David Warner and colleagues for their interest in the UK BiLEVE ${ }^{1}$ study and note their concerns regarding $\mathrm{FEV}_{1}$. In this

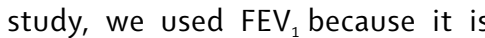
of clinical and epidemiological relevance; \% predicted $\mathrm{FEV}_{1}$ is used to grade COPD severity ${ }^{2}$ and FEV is a major predictor of all-cause mortality, even in lifelong nonsmokers. ${ }^{3,4}$ The method of lung function measurement is described in the methods and appendix of our Article; further equipment and calibration details are available on the UK Biobank website (via Field IDs 3062 and 3063;). Both FEV 1 and FVC measurements were used to refine the sampling frame to individuals with spirometry measures which met strict ATS/ERS quality guidelines. We provide absolute $\mathrm{FEV}_{1}$ values in table 1 of our Article, and absolute $\mathrm{FEV}_{1} / \mathrm{FVC}$ values in the appendix for individuals with COPD and controls.

All six of the signals associated with the extremes of $\mathrm{FEV}_{1}$ that we report also show association with COPD (GOLD stage 2-4, defined using FEV and $\mathrm{FEV}_{1} / \mathrm{FVC}$ ); (COPD association results are shown in table 1 of our Article. Warner and colleagues suggest that the signals we report could be attributed to measurement error. Any residual measurement error after the stringent quality control measures we describe would be expected to attenuate estimates towards the null, not to generate false-positive associations. Furthermore, we show replication and corroborative evidence in support of the signals we report Consistent with other common complex traits, the proportion of the variance explained by genetic variants detected in this and previous studies is modest. Generally, we expect 